\title{
CROSS-CALIBRATION OF AQUA-MODIS AND NPP-VIIRS REFLECTIVE SOLAR BANDS FOR A SEAMLESS RECORD OF CERES CLOUD AND FLUX PROPERTIES
}

\author{
Rajendra Bhatt ${ }^{a}$, David Doelling $^{b}$, Benjamin Scarino $^{a}$, Conor Haney $^{a}$, Arun Gopalan $^{a}$ \\ ${ }^{a}$ Science Systems and Applications, Inc., Hampton, VA 23666 \\ ${ }^{b}$ NASA Langley Research Center, Hampton, VA 23666
}

\begin{abstract}
The CERES measured shortwave and longwave fluxes rely on the cloud properties derived using the coincident observations from the accompanying high-resolution MODIS and VIIRS imagers. The calibration consistency is required between MODIS and VIIRS radiances to ensure that the CERES provided cloud property retrievals are temporally consistent. This paper presents multiple approaches of cross-calibrating the spectrally comparable reflective solar bands (RSB) of Aqua-MODIS and NPPVIIRS, and estimates the radiometric biases for individual band pair. The inter-comparison is performed between the Aqua-MODIS collection 6.1 level 1B and NPP-VIIRS Land PEATE V1 datasets. Radiometric biases up to $3 \%$ were estimated between the MODIS and VIIRS radiances for visible bands.
\end{abstract}

Index Terms - Cross-calibration, VIIRS, MODIS, DCC, CERES, SBAF

\section{INTRODUCTION}

The Clouds and the Earth's Radiant Energy System (CERES) instruments on board the Terra, Aqua, and S-NPP satellites have been providing global surface and top-ofatmosphere (TOA) radiative fluxes for over one and a half decade to help scientists to understand the role of clouds in the Earth's energy balance and improve climate prediction models [1]. The climate quality of the CERES data is maintained using a state-of-the-art on-board calibrator. All CERES instruments rely on simultaneous measurements from high-resolution on-board imagers, MODIS on Aqua and Terra, and VIIRS on NPP, for retrieving cloud properties, that determine scene type, which are needed to convert the observed CERES shortwave and longwave radiances into fluxes. For the reliability of such datasets, it is necessary to ensure that the imagers on different satellites are radiometrically consistent. Although MODIS and VIIRS are both equipped with a solar diffuser for their in-flight calibration, radiometric biases may still exist between them due to their finite signal-to-noise ratio, differences in the characterization of solar diffusers, instrument aging, etc.

Doelling et al [2] performed a detailed inter-consistency study of Aqua- and Terra-MODIS Collection 6 calibration utilizing multiple independent approaches, which included the use of near-simultaneous nadir overpasses (SNO) over the poles and invariant Earth targets, and found that the radiometric biases are minimal and within the specified calibration accuracy of both MODIS instruments. A similar inter-consistency analysis of MODIS and VIIRS imagers is required to ensure the radiometric consistency between the cloud retrievals from the two instruments. This paper reports radiometric biases between the matching spectral bands of Aqua-MODIS and NPP-VIIRS using three independent cross-calibration approaches. The MODIS and VIIRS radiance data used in this inter-comparison study are a reformatted subset of the MODIS Collection 6.1 level 1B product and the NPP VIIRS V1 dataset generated by the NASA Land Product Evaluation and Analysis Tool Elements (PEATE), respectively, for support of independent cloud retrievals for the CERES project. In all three methods, the spectral differences between the comparable MODIS and VIIRS channels are accounted for with scene-specific spectral band adjustment factors (SBAFs) based on hyperspectral TOA radiance measurements acquired from the SCanning Imaging Absorption SpectroMeter for Atmospheric CHartographY (SCIAMACHY) instrument as described by Scarino et al. [3].

\section{METHODOLOGY}

\subsection{ATO and DCC ray-matching}

Coincident, co-located, and co-angled radiance pairs for all comparable channels of Aqua-MODIS and NPP-VIIRS are acquired between $30^{\circ} \mathrm{N}$ and $30^{\circ} \mathrm{S}$ for all-sky tropical ocean (ATO) and deep convective clouds (DCC) scenes. For either of these ATO ray-matching or DCC ray-matching (ATORM and DCC-RM) schemes, measurements from each instrument must be gathered within 15 minutes of oneanother, with less than $40^{\circ}$ viewing zenith angle (VZA) and less than $40^{\circ}$ solar zenith angle (SZA). Conditions where the relative azimuth angle (RAZ), with respect to either instrument, is less than $10^{\circ}$ or greater than $170^{\circ}$ are avoided. In all cases, inter-satellite VZA and SZA differences must be less than $3^{\circ}$, whereas the allowable RAZ difference varies from less than $3^{\circ}$ for dark ocean scenes, less than $5^{\circ}$ for mixed ocean/cloud and darker cloud scenes, and less than $10^{\circ}$ for the brightest cloud scenes.

Both techniques have additional unique refinements to best suit their physical traits. ATO-RM focuses on capturing the entire range of Earth viewed radiances by combining clear-sky ocean, partly cloudy and overcast conditions. On 
the other hand, DCC-RM focuses on reducing the impact of the SBAF by selecting only spectrally uniform near Lambertian clouds, and therefore must employ more discriminating scene selection criteria. For ATO-RM, pixels from each instrument are averaged within a shared $50-\mathrm{km}$ (at nadir) diameter, which constitutes one ray-matched radiance pair. The spatial standard deviation must be less than $50 \%$ of the average. In case of DCC-RM, a $30-\mathrm{km}$ diameter is used, where the mean BT must be less than $220 \mathrm{~K}$ with a spatial standard deviation less than $20 \%$ of the average and a $11-\mu \mathrm{m}$ BT standard deviation less than $7.5 \mathrm{~K}$. These criteria allow for adequate distinction between the two methods with sufficient sampling, dynamic range, SBAF uncertainty, and statistical confidence. Fig. 1 shows the scatter plot of the ray-matched ATO radiance pairs acquired in July 2017 for Aqua-MODIS band $1(0.65-\mu \mathrm{m})$ and NPP-VIIRS I1 $(0.64-\mu \mathrm{m})$. A linear regression forced through zero is fitted to the data and the forced-slope is used as the cross-calibration ratio.

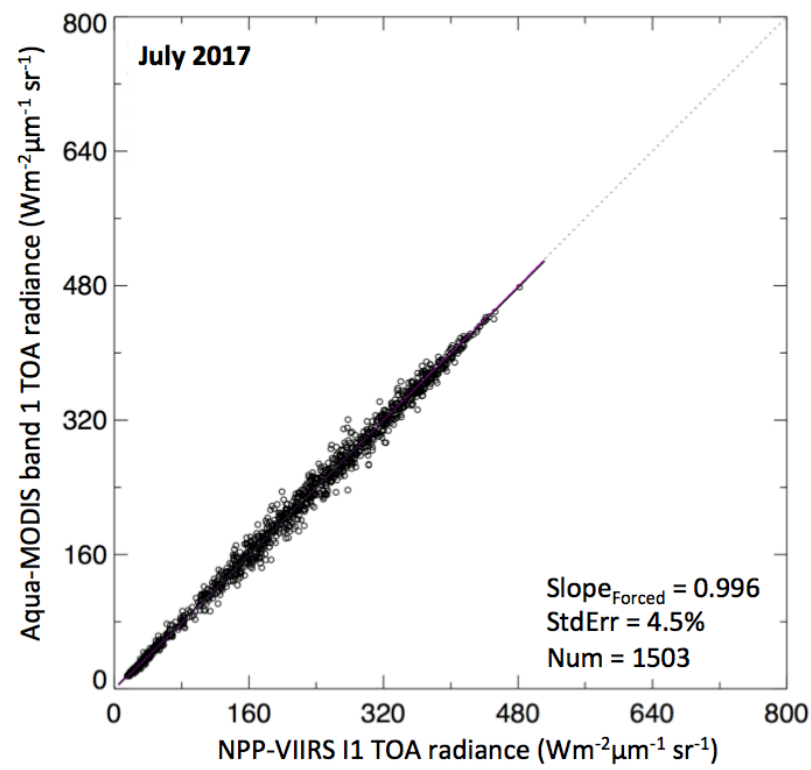

Fig. 1. ATO-RM scatter plot of Aqua-MODIS band 1 and NPP-VIIRS I1 radiances from July 2017.

\subsection{DCC invariant target (DCC-IT) method}

DCCs are an excellent invariant Earth target for vicarious calibration of satellite reflective solar bands (RSBs) [4-7]. The DCC-IT is a large-ensemble statistical approach that collectively analyzes all identified DCC pixels on a monthly basis. DCC pixels are acquired globally over the tropics ( $\pm 20^{\circ}$ latitude) for all matching spectral channels of MODIS and VIIRS using the DCC identification criteria defined by Doelling et al. [4]. For visible and near-IR (VIS-NIR) (defined here as $<1 \mu \mathrm{m}$ ) wavelengths, the DCC reflectance is mostly lambertian, and requires only small bidirectional reflectance distribution function (BRDF) corrections. $\mathrm{Hu}$
DCC BRDF is used for anisotropic corrections in VIS-NIR spectral channels [8]. The DCC reflectance in the shortwave infrared (SWIR) (defined here as $>1 \mu \mathrm{m}$ ) bands is highly affected by the ice effective diameter that produces a large seasonal cycle with its magnitude depending on the wavelength [9]. Bhatt et al. [7] illustrated that a SWIR band DCC seasonal cycle repeats annually and can be characterized with a channel-specific seasonal BRDF. The application of the seasonal BRDF can reduce the seasonal variation in the SWIR band DCC time series by up to $\sim 55 \%$ [7]. This study also incorporates channel-specific BRDFs to mitigate the temporal variation in the SWIR band DCC response of Aqua-MODIS and NPP-VIIRS. As confirmed by previous studies, the monthly probability density function (PDF) mode is more suitable to evaluate the sensor calibration stability in VIS-NIR wavelengths, whereas for SWIR bands the monthly mean DCC reflectance is preferable [4][7]. Because NPP-VIIRS and Aqua-MODIS are both in a 13:30 sun-synchronous orbit, they have consistent local time sampling of the DCC cycle, thereby avoiding any diurnal sampling differences of DCC properties. This allows a direct comparison of the monthly mode (for VIS-NIR bands) or mean (for SWIR bands) DCC response from the two instruments for computing the radiometric biases.

\subsection{PICS method}

The pseudo-invariant calibration site (PICS) method used in this paper follows the technique described by Bhatt et al. [6], where the radiometric characterization of the ground PICS is performed by constructing direction models (DMs) that define the near-nadir $\left(\mathrm{VZA}<10^{\circ}\right)$ TOA radiances as a function of cosine of the solar zenith angle (SZA). The Libya-4 $\left(28.55^{\circ} \mathrm{N}\right.$ latitude and $23.40^{\circ} \mathrm{E}$ longitude) PICS is used in this study. The channel-specific DM is constructed using the Aqua-MODIS measurements acquired over Libya4 during 2012-2017. Because the observed Libya-4 TOA radiances are slightly darker in the forward scattering direction than in the backward scattering direction, the two scattering conditions were modeled independently as described in [6]. The reference DM is then used to predict the near-nadir TOA radiances for the matching VIIRS band. The ratio of the DM-predicted VIIRS radiance and the observed VIIRS radiance provides the calibration bias between VIIRS and MODIS.

\subsection{SBAF}

The spectral response functions (SRFs) of MODIS and VIIRS RSBs exhibit some differences. SBAFs are computed for individual calibration targets to account for the spectral band differences in cross-calibration. The computation of SBAF follows the approach of Scarino et al. [3], in which the target-specific hyper-spectral footprints measured by the SCIAMACHY instrument are convolved with the SRFs of 
the two sensors that are being cross-calibrated in order to estimate pseudo-imager radiance pairs for these sensors. The SBAF is then computed as the slope of regression of the pseudo-radiance pairs. The VIIRS radiances are then adjusted to equivalent MODIS radiances using the SBAF prior to cross-calibration. The SBAF regression for spectral correction between the MODIS band 1 and VIIRS M5 channel for the PICS method using the Libya-4 target is shown in Fig. 2. The expected radiance bias due to the SRF differences between these two channels is $3.2 \%$ $(\mathrm{SBAF}=0.968)$ for Libya-4.

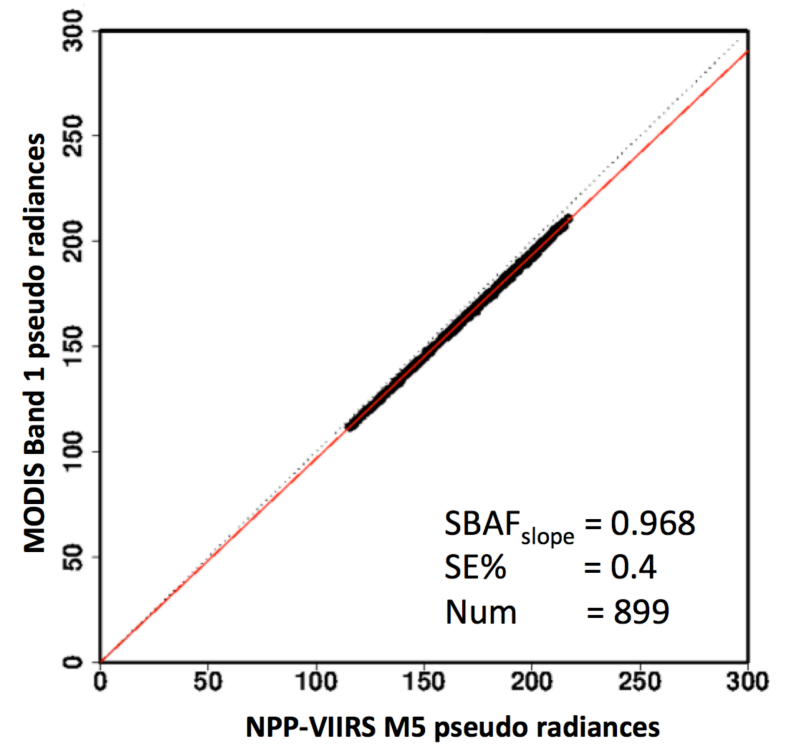

Fig. 2. The SBAF regression of SCIAMACHY-based pseudo-radiances $\left(\mathrm{Wm}^{-2} \mathrm{\mu m}^{-1} \mathrm{sr}^{-1}\right)$ for VIIRS M5 and AquaMODIS B1 over the Libya-4 desert.

\section{RESULTS}

Following the methods described above, the MODIS-toVIIRS radiance ratio is computed for each spectral band pair on a monthly basis and is trended over time. Fig. 3 shows the time series of MODIS-to-VIIRS radiance ratio for AquaMODIS band 1 and two matching VIIRS channels, I1 and M5 $(0.67-\mu \mathrm{m})$. No temporal trend is detected in any of these time series, which indicates an excellent radiometric stability of both MODIS and VIIRS datasets. The mean radiance bias after spectral corrections between VIIRS M5 and MODIS band 1 is found to be $1.4 \%, 1.9 \%, 1.6 \%$, and $1.8 \%$ using the DCC-IT, DCC-RM, ATO-RM, and PICS methods, respectively. All four methods show that the VIIRS M5 channel is brighter than MODIS band 1 . Similarly, the maximum calibration bias between VIIRS I1 and Aqua-MODIS band 1 is found to be $0.5 \%$. Calibration biases computed for the VIIRS M3 $(0.47-\mu \mathrm{m})$, M4 (0.55$\mu \mathrm{m})$, and M7 $(0.86-\mu \mathrm{m})$ channels and their corresponding matching bands in Aqua-MODIS (band 3, 4, and 2, respectively) are listed in Table 1 . Because MODIS band 2 saturates for DCC targets, the DCC-IT and DCC-RM methods are inapplicable for this channel. The observed radiance biases between MODIS and VIIRS are within 3\% for the band pairs listed in Table 1. Inter-comparison results for the remaining RSBs will be presented at the conference.

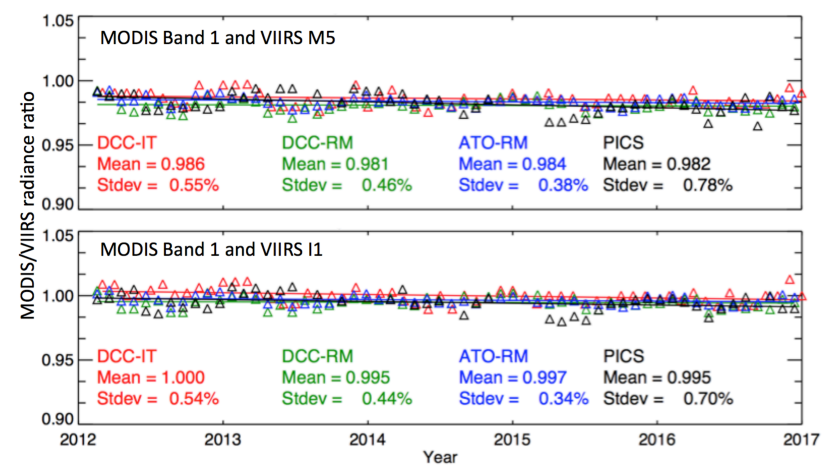

Fig. 3. Cross-calibration radiance ratios computed for AquaMODIS band 1, and VIIRS M5 (top) and I1 (bottom) bands using DCC-IT, DCC-RM, ATO-RM, and PICS approaches.

Table 1. Cross-calibration biases between MODIS and VIIRS visible and near-infrared bands.

\begin{tabular}{|l|l|l|l|l|l|}
\hline Method & B3/M3 & B4/M4 & B1/M5 & B2/M7 & B1/I1 \\
\hline DCC-IT & 0.985 & 0.982 & 0.986 & NA & 1.0 \\
\hline DCC-RM & 0.979 & 0.970 & 0.981 & NA & 0.995 \\
\hline ATO-RM & 0.992 & 0.972 & 0.984 & 0.995 & 0.997 \\
\hline PICS & 0.973 & 0.973 & 0.982 & 0.990 & 0.995 \\
\hline
\end{tabular}

\section{CONCLUSIONS}

To achieve a consistent retrieval of cloud properties across the entire CERES record, it is important to ensure the radiometric consistency between the MODIS and VIIRS instruments. In this paper, we discussed three independent approaches establishing radiometric consistency between the Aqua-MODIS and NPP-VIIRS RSBs. The ATO-RM and DCC-RM methods utilize the coincident, co-located, and co-angled radiance pairs acquired by Aqua-MODIS and NPP-VIIRS within 15 minutes. The DCC-IT is a largeensemble statistical approach that relies on seasonal BRDFs to mitigate the large seasonal cycles in the SWIR band DCC response. The PICS method does not require simultaneous measurements from MODIS and VIIRS. It strictly relies on the radiometric stability of the ground PICS and the robustness of the DMs used to characterize the PICS TOA radiance. The mean radiometric bias computed for AquaMODIS band 1 and VIIRS M5 from the multiple approaches is $1.7 \%$, whereas it is less than $0.5 \%$ for AquaMODIS band 1 and VIIRS I1 band pair. The calibration consistency of the MODIS and VIIRS visible and nearinfrared bands is found to be within 3\% after accounting for the spectral differences. The radiometric biases for other RSB pairs of MODIS and VIIRS will be presented at the conference. 


\section{ACKNOWLEDGEMENT}

The authors would like to thank the MODIS Characterization Support Team for providing the C6 to C6.1 conversion coefficients for Aqua-MODIS RSBs. This work was supported by the National Aeronautics and Space Administration Earth (NASA) Science Enterprise Office through the CERES and Research Announcement (NRA) NNH15ZDA001N, Research Opportunities in Space and Earth Science (ROSES-2015), Program Element A.34: Satellite Calibration Interconsistency Studies.

\section{REFERENCES}

[1] N. Loeb, N. Manalo-Smith, W. Su, M. Shankar, and S. Thomas, "CERES Top-of-Atmosphere Earth Radiation Budget Climate Data Record: Accounting for in-Orbit Changes in Instrument Calibration," Remote Sensing, vol. 8, no. 3, p. 182, Feb. 2016.

[2] D. R. Doelling et al., "The Radiometric Stability and Scaling of Collection 6 Terra- and Aqua-MODIS VIS, NIR, and SWIR Spectral Bands," in IEEE Transactions on Geoscience and Remote Sensing, vol. 53, no. 8, pp. 4520-4535, Aug. 2015.

[3] B. R. Scarino et al., "A Web-Based Tool for Calculating Spectral Band Difference Adjustment Factors Derived From SCIAMACHY Hyperspectral Data," IEEE Transactions on Geoscience and Remote Sensing, vol. 54, no. 5, pp. 2529-2542, May 2016.

[4] D. R. Doelling, D. Morstad, B. R. Scarino, R. Bhatt, and A. Gopalan, "The characterization of deep convective clouds as an invariant calibration target and as a visible calibration technique," IEEE Transactions on Geoscience and Remote Sensing, vol. 51, no. 3, pp. 1147-1159, Mar. 2013.

[5] D. R. Doelling et al., "The Radiometric Stability and Scaling of Collection 6 Terra- and Aqua-MODIS VIS, NIR, and SWIR Spectral Bands," in IEEE Transactions on Geoscience and Remote Sensing, vol. 53, no. 8, pp. 4520-4535, Aug. 2015.

[6] R. Bhatt, D. Doelling, A. Wu, X. Xiong, B. Scarino, C. Haney, and A. Gopalan, "Initial Stability Assessment of S-NPP VIIRS Reflective Solar Band Calibration Using Invariant Desert and Deep Convective Cloud Targets," Remote Sensing, vol. 6, no. 4, pp. 2809-2826, Mar. 2014.

[7] R. Bhatt, D. Doelling, B. Scarino, C. Haney, and A. Gopalan, "Development of Seasonal BRDF Models to Extend the Use of Deep Convective Clouds as Invariant Targets for Satellite SWIRBand Calibration," Remote Sensing, vol. 9, no. 10, p. 1061, Oct. 2017.

[8] Y. Hu, B. A. Wielicki, Ping Yang, P. W. Stackhouse, B. Lin and D. F. Young, "Application of deep convective cloud albedo observation to satellite-based study of the terrestrial atmosphere: monitoring the stability of spaceborne measurements and assessing absorption anomaly," IEEE Transactions on Geoscience and Remote Sensing, vol. 42, no. 11, pp. 2594-2599, Nov. 2004.
[9] S. Platnick, J.Y. Li, M.D. King, H. Gerber, and P.V. Hobbs, “A solar reflectance method for retrieving the optical thickness and droplet size of liquid water clouds over snow and ice surfaces," $J$. Geophys. Res., vol. 106, pp. 15185-15199, Jul. 2001. 